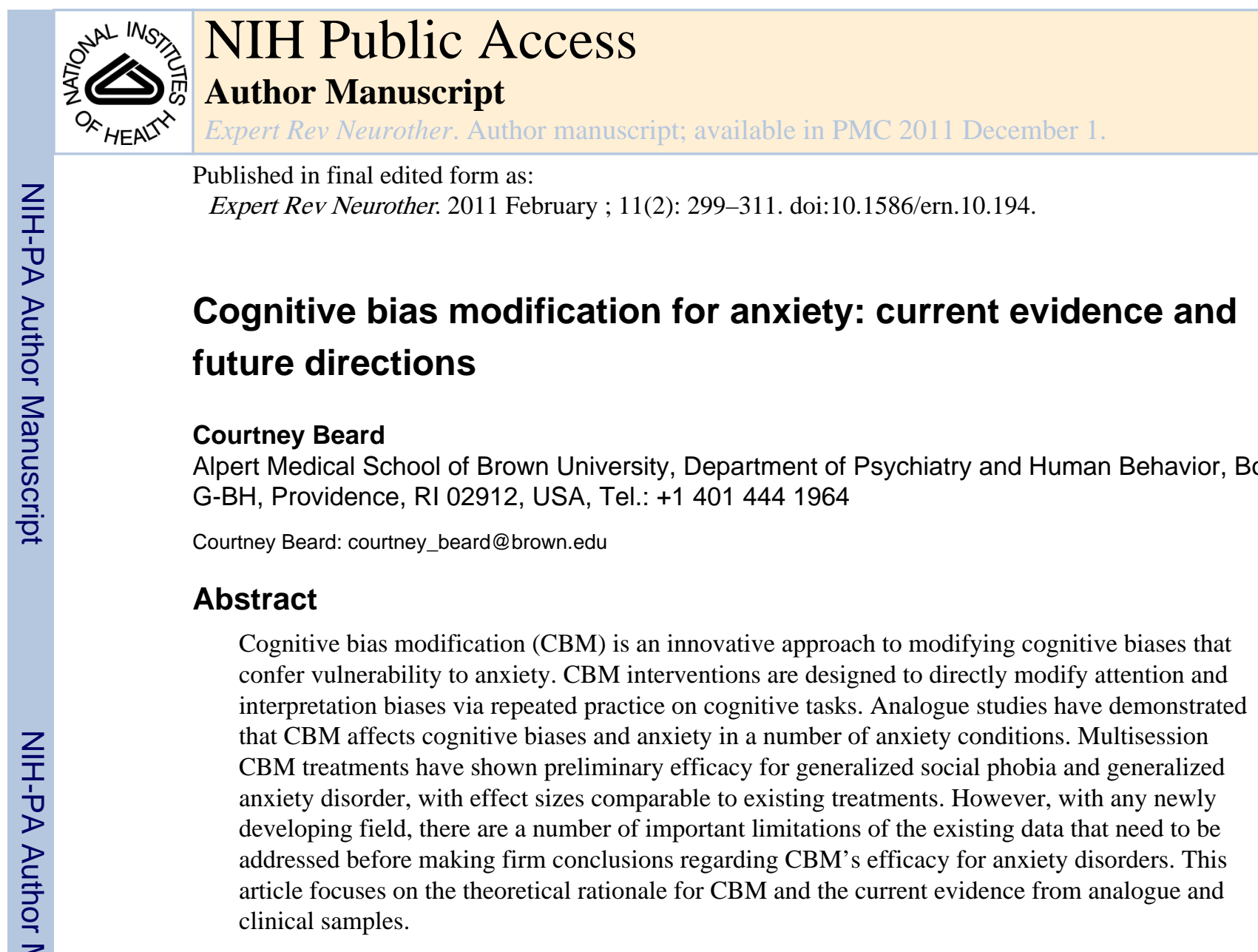

\title{
Keywords
}

anxiety disorders; attention bias; attention training; cognitive bias modification; interpretation bias; review; treatment

\section{Anxiety disorders}

Anxiety disorders are the most common type of psychiatric disorder. Recent estimates suggest that approximately $30 \%$ of the US population will suffer from an anxiety disorder in their lifetime [1]. In addition to being highly prevalent, anxiety disorders are associated with significant functional impairment. Compared with the general population, individuals with anxiety disorders experience poorer quality of life [2,3], educational and occupational impairment [4,5] and higher suicide rates [6,7]. Moreover, anxiety disorders place a burden on society through lost work productivity, disability and increased healthcare utilization $[8,9]$. Thus, anxiety disorders are a significant public health problem and require effective, acceptable and accessible treatments.

There are a number of efficacious treatments available for anxiety disorders. Pharmacologic treatments, particularly selective serotonin reuptake inhibitors/serotonin-norepinephrine

\section{(C) 2011 Expert Reviews Ltd}

For reprint orders, please contact reprints@expert-reviews.com

Financial \& competing interests disclosure

Courtney Beard's time and effort was supported in full by an NIMH NRSA postdoctoral fellowship (F32 MH083330). The author has no other relevant affiliations or financial involvement with any organization or entity with a financial interest in or financial conflict with the subject matter or materials discussed in the manuscript apart from those disclosed.

No writing assistance was utilized in the production of this manuscript. 
reuptake inhibitors, alleviate anxiety symptoms in the majority of patients. However, effect sizes are small-to-medium, and approximately $40 \%$ of patients do not achieve remission with pharmacotherapy $[10,11]$. Psychosocial treatments, particularly cognitive-behavioral therapy (CBT), have also demonstrated efficacy. Meta-analyses suggest that CBT alleviates anxiety with medium-to-large effect sizes, depending on the disorder and study characteristics $[12,13]$. Although effective treatments exist, many patients with anxiety disorders do not access these treatments for a variety of reasons. For example, untreated individuals with an anxiety disorder report concerns about side effects and not believing in the use of medication for emotional problems [14]. Barriers to accessing CBT include a lack of trained therapists, and therapist and patient unwillingness to engage in exposure therapy [15], which is a key component of CBT.

Thus, while treatments for anxiety have improved substantially over the past few decades, they leave much room for improvement in their accessibility and magnitude of response. There are a number of mechanisms involved in anxiety disorders that are potential targets for new treatments. After presenting the theoretical and empirical rationale for targeting cognitive biases in treatment, this article focuses on one promising novel treatment for anxiety disorders, cognitive bias modification (CBM). At the time of preparing this article, the author is aware of four excellent reviews that overlap to various degrees with the current review. Please see Bar Haim [16], Browning et al. [17] and Hakamata et al. [18] for reviews specifically focused on cognitive bias modification for attention (CBM-A). Taylor and Amir review strategic attentional control treatments in addition to CBM-A [19].

\section{Why target cognitive biases?}

Individuals with anxiety disorders tend to selectively process threat-relevant information in their environment. For example, when speaking in a group, a socially anxious individual is likely to attend to threatening facial expressions (e.g., anger and disgust) rather than neutral expressions. That individual may also be more likely to interpret a negative facial expression as indicative of the conversation partner's disgust with the speaker rather than the content of the conversation. As there is usually more information than cognitive resources available to process the information, this habit of selectively attending (attention bias) and interpreting (interpretation bias) creates a vicious cycle in which an ambiguous world is experienced as threatening. Cognitive models of anxiety propose a number of cognitive biases and vulnerabilities that maintain anxiety. However, this article focuses on two primary cognitive biases - that is, attention and interpretation - implicated in anxiety disorders and targeted by CBM.

\section{Attention bias}

The term 'attention' can refer to a wide range of cognitive processes. In the informationprocessing field, attention bias usually refers to the tendency to selectively attend to threat stimuli, even when those stimuli are irrelevant to current goals and/or when it is in competition for attention with nonthreat stimuli. Attention bias is often measured with visual stimuli presented very quickly (e.g., $500 \mathrm{~ms}$ ) in order to tap this initial stage of information processing. Currently, the most commonly used measure of attention bias is the dot probe task, originally developed by MacLeod and colleagues [20]. In one version of the dot probe task (Figure 1), two stimuli are presented on a computer monitor simultaneously for $500 \mathrm{~ms}$. One of the stimuli is neutral (e.g., the word 'chair'), and one is threat-relevant (e.g., the word 'disease'). Then, the stimuli disappear, and a probe (e.g., the letter ' $E$ ' or ' $F$ ') appears in the previous location of either the neutral or threat stimulus. Probes replace neutral and threat stimuli with equal frequency. A participant's task is to identify the probe as quickly and accurately as possible by pressing a corresponding button. Biased attention toward threat is inferred from faster reaction times to identify probes replacing threat stimuli compared with 
neutral stimuli. An overall attention bias score can be calculated from reaction times on the different types of trials.

Bar-Haim's meta-analysis of 172 studies revealed that attention bias and anxiety are reliably associated with an average effect size of Cohen's $d(d)=0.45$ (confidence interval $[\mathrm{CI}]$ : $0.40-0.49$ ). Anxious individuals exhibit an attention bias across a range of populations (adults and children), stimuli (words and pictures) and stimuli presentation durations (subliminal and supraliminal) [21]. Thus, the association between anxiety and attention bias is a reliable finding in experimental psychopathology research.

Prospective studies have extended this correlational evidence by demonstrating that attention bias is a vulnerability factor for developing anxiety in response to stress. In one study, attention bias at baseline predicted anxiety and dysphoria in participants learning of a cancer test result 8 weeks later [22]. Moreover, attention bias was a stronger predictor of emotional responses than self-report measures of distress at baseline. This effect has been replicated [23]. In addition, a recent study demonstrated that attention bias at baseline predicted salivary cortisol response to laboratory and natural stressors 4 months later better than self-report measures of anxiety and neuroticism [24]. In summary, cognitive models and empirical data from correlational and prospective studies suggest that attention bias plays a significant role in the development and maintenance of anxiety and should be targeted in treatment.

\section{Interpretation bias}

Anxiety disorders are also characterized by interpretation bias, the tendency to interpret ambiguous or mildly negative cues in a negative or catastrophic manner. Researchers have developed various self-report and reaction-time measures to assess interpretation. For example, some assess interpretation by comparing participants' reaction time in responding to threat interpretations versus non-threat interpretations of ambiguous text as they are completing a specific task. It is assumed that participants will respond more quickly to interpretations that are consistent with their own immediate, 'online' interpretation. For example, Hirsch and Mathews compared interpretation bias in individuals with high and low interview anxiety [25]. Participants read narrative texts describing interviews that included critical ambiguous sentences on which participants had to make a decision about the final word. The final word resolved the ambiguity in either a positive or negative manner. For example, after reading the incomplete sentences: 'you tell the interviewer your idea and she seems to be ... interested,' or 'you wonder if, when you are in the interview, all your preparation will be ... forgotten,' participants were asked to indicate as quickly as possible whether the probe word was grammatically correct (Exp 1) or whether it was a real word or not (i.e., lexical decision task, Exp 2). These researchers then compared response latencies to make decisions regarding negative versus positive probe words. In this paradigm and others, anxious individuals exhibit biased interpretation compared with nonanxious individuals [26-30].

A number of reviews suggest that interpretation bias is a robust phenomenon associated with anxiety in both children and adults and across a wide range of paradigms (e.g., scenarios, homographs and self-report) and stimuli (words and videos) [31,32]. Moreover, several prospective studies have demonstrated that negative interpretation of intrusive thoughts at baseline predicts post-traumatic stress disorder and obsessive-compulsive disorder (OCD) symptom severity at follow-up in both children [33] and adults [34-36]. Thus, similar to attention bias, interpretation bias is an important vulnerability factor that should be targeted in treatment. 


\section{Treatments targeting cognitive biases}

Given the important role cognitive biases play in anxiety disorders, several treatments have been developed to target these processes. For example, mindfulness-based and attention/ meta-cognitive training approaches teach patients to better control their attention through specific exercises [37,38]. CBTs target distorted interpretations via cognitive restructuring and behavioral experiments. Indeed, anxious individuals who respond to successful treatments show a significant reduction in attention and interpretation biases, while nonresponders do not [39-42].

The aforementioned approaches rely on verbal dialogue and explicit instruction to change cognition. They facilitate strategic control over attention and interpretation. However, cognitive biases are often automatic in nature and not always under volitional control. They can be thought of as habits, and may be more efficiently and directly modified via repeated experiential practice on tasks that require rapid processing. CBM is a novel approach designed to modify cognitive biases in such a manner.

Koster et al. provide an excellent working definition for the emerging field of CBM [43]. They identify two key features of CBM: first, "the direct target of change in each case is a cognitive bias known to characterize a clinical disorder, a clinically relevant symptom, or a personality trait associated with vulnerability to clinical dysfunction"; and second, "the method of manipulating the target cognitive bias has not principally relied on instruction but instead has involved extensive practice on a cognitive task designed to encourage and facilitate the desired cognitive change." Thus, CBM procedures may alter cognitive biases through a more implicit, experiential process compared with the explicit, verbal process of psychotherapy. Although completion of a CBM task is volitional, the processes targeted in the task are not usually considered to operate under volitional control, and participants are not usually aware of the purpose of the CBM task.

\section{Cognitive bias modification - attention}

To date, the most commonly used CBM-A task is a modified version of the dot probe task, described previously as a method to assess attention bias. When using the dot probe task to assess attention, probes replace neutral and threat stimuli with equal frequency. To modify attention, researchers alter the contingency between the location of probes and threat stimuli. For example, in order to guide attention away from threat stimuli, probes should always replace neutral stimuli.

While most CBM-A studies have used versions of the dot probe task, Dandeneau and colleagues developed a different CBM-A intervention based on a visual search task [44]. The task requires individuals to repeatedly identify the location of a smiling face among a matrix of angry faces. The task is designed to improve an individual's ability to inhibit attention toward rejection and facilitate attention toward acceptance. For both the dot probe and face in the crowd paradigms, completing hundreds of trials in which attention is repeatedly disengaged from threat stimuli is designed to reduce attention bias for threat. Cognitive theories predict that successful reduction of attention bias should decrease an individual's vulnerability to anxiety.

\section{Analogue samples}

To date, most research has tested whether CBM-A can modify attention and anxiety in nonanxious individuals. In analogue studies, participants are randomly assigned to an 'attend threat' condition, in which probes usually replace threat stimuli, or an 'attend neutral' condition, in which probes usually replace neutral or positive stimuli. Participants are not given any information about the study hypothesis or the purpose of the computer task. Study 
designs usually comprise of a single experimental session during which participants complete a pre-assessment of attention and anxiety, a training block comprising of hundreds of dot probe trials, a postassessment of attention and anxiety, a stressor (e.g., worry induction, anagram task or impromptu speech) and a poststressor assessment of anxiety (Figure 2). Thus, the typical study design assesses the direct effect of CBM-A on both attention and anxiety (e.g., post-training assessment), and on anxiety vulnerability in response to stress (e.g., poststressor assessment). Alternatively, rather than include a laboratory stressor, some studies have examined CBM-A's effects on the response to natural life stressors (e.g., telemarketer job performance [45], final exams [45] and leaving one's home country for school [46]).

Using these various designs, a number of studies have demonstrated that it is possible to induce an attention bias toward threat in nonanxious adults and children, similar to that observed in anxious individuals [47-51]. As expected, inducing an attention bias toward threat increased anxiety response to stressors compared with a condition that induces a neutral bias. For example, in the classic study by MacLeod et al., participants completed an anagram stress task following attention training [47]. They were told that their videotaped performance was unusually poor and would be used for demonstration purposes in psychology classes. In response to this stressor, participants in the neutral condition reported significantly less negative mood compared with the threat condition.

The evidence suggests that CBM-A does not appear to directly affect anxiety or mood, as groups do not differ on emotional measures directly after training. Rather, effects only emerge in response to stress, suggesting that CBM-A is altering cognitive vulnerability to anxiety, rather than manipulating mood. These findings suggest that cognitive biases play a causal role in anxiety vulnerability, further supporting attention bias as a treatment target.

Researchers have used similar designs to test whether CBM-A can affect attention in anxious samples (Table 1). For ethical reasons, these studies do not typically induce a threat bias, but rather use a control group. Specifically, an 'attend neutral' condition is usually compared with a control condition, in which probes replace threat and neutral stimuli with equal frequency. Thus, the control condition is identical to the traditional dot probe task used to assess attention. One session of CBM-A has been found effective for modifying attention bias and anxiety in individuals with high anxiety/worry [52,53], public speaking anxiety [54] and OCD contamination fears [55], but not spider fear [56].

It is promising, and perhaps surprising, that a single, brief (less than $1 \mathrm{~h}$ ) session of CBM-A affects cognitive processing and anxiety vulnerability. Given the potential clinical benefit of CBM-A, several studies have examined the effect of multisession CBM-A interventions on symptoms of anxiety. Repeated performance of the tasks is hypothesized to produce changes in attention that generalize to changes in attentional processes in an individual's real world and have a meaningful impact on anxiety. For example, See and colleagues administered 15 daily sessions of CBM-A that were delivered online in participants' homes [46]. Participants comprised of high school students preparing to move to a foreign country for college. Compared with a control group that had no training, CBM-A resulted in lower anxiety scores on moving day. Moreover, change in attention bias from baseline to postassessment (prior to moving day) mediated the relationship between group and change in anxiety state from baseline to moving day. Thus, CBM-A may buffer against negative emotional responses to life stressors in healthy individuals.

Multisession protocols have also been examined in highly anxious samples. Li and colleagues tested CBM-A in individuals with high social anxiety [57]. After seven daily sessions, the CBM-A group demonstrated a reduction in attention bias compared with a 
control group. However, only one out of three self-report measures revealed group differences in social anxiety symptoms. Another study administered five weekly sessions of CBM-A to high worriers, some of whom met diagnostic criteria for generalized anxiety disorder (GAD) [58]. Compared with a control condition, CBM-A resulted in significant reductions in attention bias and a composite, self-report anxiety and depression score.

\section{Clinical samples}

Given the promising findings in analogue samples, researchers have begun testing multisession CBM-A treatments for diagnosed anxiety disorders. To date, all studies have been double-blind, placebo-controlled trials. This is a unique methodological advantage over other psychosocial treatment trials, in which typically only assessors are unaware of participants' condition assignment. To date, three CBM-A treatment protocols have involved eight 15-30 min sessions completed twice per week (4 weeks total intervention duration). Participants did not receive any psychoeducation, treatment rationale or clinician contact.

Generalized social phobia-There have been two randomized controlled trials (RCTs) of CBM-A for individuals diagnosed with generalized social phobia (GSP). Schmidt and colleagues randomized 36 patients with GSP to CBM-A or placebo (traditional dot probe task) [59]. The dot probe task presented disgust and neutral faces. Groups began to diverge in social anxiety symptoms at post-treatment, and they were significantly different at a 4month follow-up assessment on both clinician (Liebowitz Social Anxiety Scale) and selfreport measures (Social Phobia and Anxiety Inventory-Social Phobia Subscale). Betweengroup effect sizes $(\mathrm{d}=0.35-0.41)$ fell in the lower end of the range of existing psychosocial and pharmacological treatments for GSP.

Amir and colleagues utilized the same CBM-A materials and protocol as Schmidt et al. and replicated the effects on social anxiety symptoms (between group effect sizes, $\mathrm{d}=0.69$ 1.59), including maintenance of gains at 4-month follow-up [60]. Moreover, they included an independent measure of attention bias (i.e., spatial cueing task with words rather than the dot probe task with faces) and demonstrated that changes in attention generalized to a different measure of attention bias and to a different type of stimulus. Finally, it was demonstrated that changes in social anxiety were mediated by changes in attention bias.

Generalized anxiety disorder-To date, there has been one RCT of CBM-A for individuals diagnosed with GAD. Amir and colleagues randomized 29 treatment-seeking individuals with GAD to CBM-A or placebo [61]. Given the heterogeneity of worry domains in GAD, the dot probe task presented personalized stimuli rather than the typical standard stimuli across participants. To this end, participants rated the valence of words at pretreatment, and only those words rated most negatively (paired with neutral words) were used for each participant. Participants completing CBM-A reported less anxiety than the placebo group on both self-report (Spielberger State Trait Anxiety Inventory) and clinicianrated (Hamilton Anxiety Rating Scale) measures, but not on the Penn State Worry Questionnaire. Between-group effect sizes for changes in anxiety $(\mathrm{d}=0.72-0.88)$ were in the range of existing treatments for GAD [62]. Results for effects on depression were mixed, with significant changes on the Beck Depression Inventory, but not the interviewer-rated Hamilton Rating Scale for Depression. Change in attention bias was found to mediate changes in anxiety, as measured by the Hamilton Anxiety Rating Scale. It is unclear for how long the effects lasted, as this study did not include a follow-up.

In summary, two independent trials testing CBM-A for GSP suggest that an eight-session protocol is efficacious in reducing symptoms and maintaining gains for at least 4 months. 
One trial testing a personalized CBM-A for GAD suggests potential efficacy, but requires independent replication and follow-up data.

A recent meta-analysis provides further support for the clinical use of CBM-A. Hakamata and colleagues reviewed studies comparing CBM-A with a control condition [18]. Results revealed a medium effect size of CBM-A on anxiety overall $(\mathrm{d}=0.61 ; \mathrm{CI}=0.42-0.81)$ with a larger effect specifically in clinical patients $(\mathrm{d}=0.78 ; \mathrm{CI}=0.38-1.20)$. The double-blind, placebo-controlled design of all the studies suggests that current evidence is probably not due to group differences in demand effects, expectancy or credibility, and rather only reflects the manipulation of probe locations. Existing effect size estimates may potentially be enhanced given the lack of rationale provided to participants, lack of therapist contact and brevity of these initial protocols.

\section{Cognitive bias modification - interpretation}

The most commonly used cognitive bias modification for interpretation (CBM-I) task is the Mathews and Mackintosh scenario paradigm, which presents participants with three-line scenarios that remain ambiguous until the final word [63]. This final word is usually a fragment that resolves the ambiguity in either a negative or benign manner. Participants are asked to solve the fragment as quickly as possible:

"Your partner asks you to go to an anniversary dinner that their company is holding. You have not met any of their work colleagues before. Getting ready to go, you think that the new people you will meet will find you (boring/friendly)."

Participants then answer a comprehension question that reinforces the forced interpretation. A similar task uses ambiguous homographs (e.g., ruin) and word fragments (ancient vs bankrupt), rather than scenarios. In both versions of this task, the word fragments almost always resolve the scenarios/homographs in a benign manner to create a more benign interpretation style. Participants typically complete approximately 100 trials. Changes in interpretation are measured with a 'recognition task' that presents new scenarios that remain ambiguous. After reading these test scenarios, participants then rate the similarity of four sentences to the original scenario. One sentence represents a positive interpretation of the original scenario, and one represents a negative interpretation. In addition, in order to assess whether the task in fact modified interpretation rather than inducing a response bias, two foil sentences (one positive and one negative in valence) that are not possible interpretations of the original scenario are also rated.

\section{Analogue findings}

To date, more than ten experiments have demonstrated that it is possible to modify interpretation in healthy and anxious individuals using CBM-I and that the effects last for at least $24 \mathrm{~h}$ (Table 2) [64-75]. The effects of CBM-I on emotion are more complicated than in CBM-A. First, many studies have demonstrated that a benign training session, compared with a negative training session or control task, leads to differences in anxiety and mood [63,66,67,70-81]. In contrast to CBM-A, CBM-I may have direct effects on emotion in addition to affecting emotional vulnerability. For example, in the classic study by Mathews and Mackintosh, multiple experiments demonstrated that participants completing a positive training session reported a reduced anxiety state immediately following training compared with a negative training session [63].

On the other hand, several of these studies have demonstrated CBM-induced effects on emotion in response to a stressor task [66,72,77,80-82]. For example, in a study by Wilson and colleagues, participants were trained to interpret homographs in either a threatening or benign manner [82]. Groups did not differ in anxiety immediately following training. 
Following training, participants viewed video clips of accidents and rescue operations. Participants in the threat condition reported significantly increased anxiety from pre- to poststressor, whereas the neutral condition did not. Finally, at least two studies have not revealed CBM-I effects on anxiety $[69,83]$.

At least four studies have tested multisession CBM-I protocols in double-blind, controlled trials. One study examined four sessions of CBM-I in adults with high trait anxiety [79]. Results revealed that 2 weeks of CBM-I resulted in more positive interpretations and reduced trait anxiety compared with a test-retest control. In a second study, adults with high trait anxiety completed eight daily sessions at home of either CBM-I or a placebo task [84]. Positive findings emerged for measures of state and trait anxiety, but not for social anxiety or stress vulnerability. A third study examined a multisession protocol in socially anxious children aged 10-11 years [72]. After completing three sessions over 1 week, children showed more positive interpretations and less social anxiety compared with a test-retest control.

A fourth multisession study [76] tested a different CBM-I task based on the Word Sentence Association Paradigm (WSAP) [28]. In that task, a word appears for $500 \mathrm{~ms}$ that represents either a negative interpretation (e.g., 'embarrassing') or a benign interpretation (e.g., 'funny') of an ambiguous sentence that follows (e.g., 'people laugh after something you said'). Participants indicate if they think the word and sentence are related or not. In order to modify interpretation, participants receive feedback about their responses. They receive positive feedback ('you are correct!') when they endorse benign interpretations or reject negative interpretations of the ambiguous sentences. They receive negative feedback ('you are incorrect') when they endorse negative interpretations or reject benign interpretations. This feedback contingency is intended to reinforce a benign interpretation bias and extinguish the negative interpretation bias associated with anxiety. After eight twice-weekly sessions, this CBM-I task successfully decreased negative interpretations, increased benign interpretations, and decreased social anxiety symptoms compared with the placebo condition. Moreover, changes in benign interpretation mediated CBM-I's effect on social anxiety.

\section{Clinical findings}

Testing of CBM-I in clinical samples lags behind that of CBM-A. To date, there are no published findings for multisession CBM-I treatments for diagnosed anxiety disorders. The clinical efficacy of CBM-I has only been examined in patients with GAD in a single-session experiment [77]. In that study, GAD patients completed both the scenario and homograph CBM-I tasks. After a single session, the benign group exhibited changes in interpretation on two different tasks compared with the control. Moreover, the benign group experienced fewer negative thought intrusions during a breathing focus task. In summary, data from the above study and several analogue studies suggest that CBM-I may have clinical utility. However, conclusions regarding the efficacy of CBM-I in clinical populations await data from future RCTs.

\section{Safety \& patient acceptability}

No published studies specifically described any safety concerns or adverse events related to CBM. This is not surprising as one might assume that CBM would not cause any side effects or serious adverse events because it merely involves presenting people with stimuli they might see in everyday life. Attrition rates provide one source of data regarding acceptability. In the RCTs reviewed, dropouts were quite low, ranging from 0 to $8 \%$. However, only one of these studies utilized the Consolidated Standards of Reporting Trials (CONSORT) flowchart [60], and all studies provided participants with compensation for attending 
sessions. Thus, attrition rates should be interpreted in this context. We do not know the feasibility or acceptability of delivering CBM without monetary incentives or in the 'real world'.

Data regarding the credibility of CBM can be inferred from participants' guesses about their treatment condition. A few researchers asked participants at the end of the study whether they believed they completed a treatment or placebo protocol. In CBM-A, only one out of 18 participants (6\% [59]) correctly guessed that they received a treatment compared with six out of 13 (46\% [76]) in CBM-I. While CBM-I appears to be more face valid, a significant proportion of participants receiving either type of CBM believed they were receiving a placebo. Thus, although participants receiving CBM exhibited decreased anxiety symptoms compared with placebo, they were either not aware of these changes in anxiety, and/or they did not attribute these changes to CBM. This discrepancy is likely due to previous CBM protocols' lack of treatment rationale and the low face validity of CBM, particularly CBMA. This lack of credibility suggests that CBM may require improvements before it will be widely accepted by stakeholders.

Preliminary unpublished studies that have explicitly assessed acceptability and satisfaction with CBM echo these concerns. Beard and colleagues have conducted two studies to examine attitudes toward CBM-A and CBM-I [85]. The first study comprised of qualitative interviews with ten anxious primary care patients. A follow-up study examined the feasibility and acceptability of a multisession CBM treatment in real-world settings. That randomized trial did not provide compensation for session attendance, provided a brief treatment rationale and delivered CBM in primary care clinics, as well as research offices. To date, 32 individuals with GSP have been assigned to either a combination CBM-A/CBMI or placebo. For participants who attended at least one session, attrition rates (18.8\%) were twice the rates of previous CBM trials, but within the range of existing treatments. Preliminary data from the Client Satisfaction Questionnaire (CSQ) suggest that CBM is acceptable to patients. Items on the CSQ are rated from 1 (dissatisfied) to 4 (satisfied). Participants' mean ratings for all items were three or greater (e.g., 'mostly satisfied').

Qualitative data from both studies revealed a range of experiences. Only two participants understood the purpose of the CBM-A task. Many participants experienced the CBM-A dot probe task as 'repetitive', 'weird' and 'confusing'. In particular, patients expressed the need to understand how the task was supposed to help with anxiety. This will be an important issue to resolve given that most previous studies have not provided any rationale to participants. Some participants found the faces in the CBM-A task to be 'creepy' and looked away from the computer monitor while the faces appeared, and other participants thought the faces were 'funny', 'exaggerated' and 'outdated'. Most participants preferred the CBM-I task and believed it improved their self-awareness of their negative mental habits and changed how they viewed situations in their daily life.

Most participants found the eight twice-weekly session schedule acceptable and would have even preferred 12 sessions. Interviews also revealed a desire for nonpharmaceutical treatment options and an interest in having a computerized treatment that is transportable and repeatable. In summary, preliminary data suggest that CBM is acceptable to patients, but also that there is room for improvement in the task stimuli, as well as treatment engagement and credibility. This will be an important area for future research, as it has direct implications for the dissemination of CBM.

\section{Mechanism of action}

Several studies have demonstrated that changes in the specific cognitive bias targeted by CBM mediated the relationship between group assignment and changes in anxiety 
symptoms $[46,52,60,61,76,86]$. Recent studies also highlight the important role of attentional control in the expression of attention bias toward threat. Specifically, individual differences in the ability to control attention moderate the relationship between attention bias and anxiety, such that anxious individuals with good attentional control do not exhibit attention bias [87]. Thus, CBM-A may be improving attentional control overall, rather than targeting the stimulus-driven (bottom-up) components of attention bias toward threat. Supporting this hypothesis, Koster and colleagues found that CBM-A influenced late (1500 $\mathrm{ms})$ rather than early $(30 \mathrm{or} 100 \mathrm{~ms})$ stages of threat processing [88]. In addition, recent neuroimaging data revealed that CBM-A altered lateral frontal activation to emotional stimuli, reflecting controlled processing [48]. Further studies are needed to examine CBMA's effect on behavioral (e.g., standard flanker task) and neuroimaging measures of attentional control.

Cognitive bias modification for interpretation studies have examined a number of potential processes underlying the scenario training task's effects. For example, researchers have demonstrated that CBM-I does not simply create a response bias consistent with the training condition [89]. Recent studies suggest that the repeated exposure to valenced stimuli in CBM-I may directly affect state anxiety, whereas changes in interpretation resulting from CBM-I may directly affect trait anxiety [90]. Importantly, changes in interpretation endure despite fluctuations in mood [71,90]. Thus, changes in interpretation observed in CBM-I studies likely represent true changes in interpretation, rather than artifacts of changes in mood. In addition, studies have demonstrated that the active generation of interpretations (e.g., completing a word fragment) are necessary for changes in anxiety to occur [63,91]. Finally, imagery probably plays an important role in the efficacy of CBM-I. A number of studies have demonstrated that encouraging participants to imagine themselves in the scenarios produces superior effects to more verbal-based instructions [92].

\section{Limitations of current evidence}

Although the potential clinical utility of CBM is quite exciting, the existing data do not address a number of limitations. First, the majority of the evidence of CBM's effect on cognitive bias and anxiety relies on analogue samples and brief (one session) experiments.

The field is in need of RCTs to test treatment protocols in clinical samples. A related issue is that all existing RCTs represent researchers' initial pilot studies rather than large-scale RCTs. Therefore, they comprise of relatively small samples (subject numbers ranging from 29 to 44). Effect sizes from small studies are unreliable [93]; thus we await the results of larger, definitive trials. Moreover, with the exception of one study, none of the studies followed the CONSORT guidelines and most did not identify a primary outcome measure. It is commendable that some of these initial RCTs included a follow-up assessment. However, for the aforementioned reasons, more evidence regarding the duration of effects for such a brief treatment are also needed.

Second, few studies have included independent measures of cognitive bias. That is, most studies assessed changes in cognitive bias using the same type of task used to modify cognitive bias. Although this provides a necessary manipulation check and allows initial tests of mechanisms of action, it does not provide information about changes in cognitive processing beyond the confines of a particular paradigm. Of those studies that did include independent measures, results have been mixed regarding generalization of cognitive change, with some studies finding generalization (CBM-A [44,45,54,60,94]; CBM-I $[65,71,77,79])$ and others not (CBM-A [95]; CBM-I [74,81,84,96]). The lack of generalization found in some studies could suggest that CBM may not change cognition in real-world situations. Indeed, it is extremely difficult to measure an individual's attention and interpretive processes in the real world, and this has yet to be done in CBM studies. 
However, findings that observed changes in cognitive bias in the laboratory mediate CBM's effect on clinical symptom severity and behavior provide some indication that cognitive changes may be generalizable to real-world situations. Clearly, more research is necessary to determine the boundaries of cognitive changes following CBM. Finally, data on patient acceptability and satisfaction with CBM are lacking. Although efforts are underway to address this gap, more researchers need to consider this important outcome in future endeavors.

\section{Future directions}

Cognitive bias modification research has grown exponentially since the first studies appeared approximately a decade ago and there is plenty of room for continued growth. To address the limitations of the current available data, we need CBM studies comprising of: first, large RCTs with intent-to-treat analyses, specified a priori primary outcomes and follow-up data; second, tests of proposed mediators and moderators; third, data on patient acceptability and satisfaction; and fourth, independent measures of attention and interpretation in order to examine the generalization of cognitive changes, including neural correlates. Including multiple behavioral and neural measures of cognitive processing may also provide information about whether CBM affects a genuine cognitive change or a conditioned response. Since the vast majority of CBM studies assessed cognitive changes with the same type of task used in training, it is impossible to know whether participants learned a task-specific response or if attention and interpretation processes genuinely changed.

In addition, there are a number of other important cognitive vulnerabilities in emotional disorders that may be targeted by CBM. For example, promising results have been found for modifying memory in depression [97]. In addition, Clerkin and Teachman developed a CBM-I for individuals with high OCD symptoms in which scenarios focused on pathological beliefs in OCD (e.g., need for control over thoughts, importance of thoughts and intolerance of uncertainty) [98]. Thus, scenarios focused on the interpretation of the meaning of an intrusive thought ('having this thought means I'm crazy/normal'), rather than the content of the thought. Such extensions of CBM-I may be adapted for other disorders with similar maladaptive beliefs, such as GAD. Researchers hoping to develop CBM-I tasks for clinical populations might consider approaches such as this in order to cause meaningful change to maladaptive cognitive processing, in addition to targeting the interpretation of specific situations. Future research will reveal whether it is possible to modify these additional cognitive processes in clinical populations, as well as whether targeting multiple vulnerabilities simultaneously is superior to targeting a single bias.

There are a number of issues involved in determining the ideal protocol. First, identifying both the ideal and minimum dosage schedule for therapeutic benefit will be important. Various multi-session protocols have been tested with positive results (e.g., daily vs weekly administration). However, only one protocol (eight sessions over 4 weeks) has been tested in clinical samples. These protocols produced effect sizes within the range of existing treatments in less time and with no clinician contact. It is possible that increasing the dosage of CBM may further enhance the magnitude of effects, but this hypothesis awaits testing.

Another interesting future direction will be development of new CBM paradigms or combinations of paradigms that may address the limitations of current CBM tasks (e.g., lack of generalization and low credibility). Although the dot probe task is the most commonly used CBM-A task, a number of studies have used a face in the crowd paradigm. In each of these tasks, acquiring an attention bias away from threat will facilitate performance. However, each task may be modifying different components of attention bias, and we do not 
know if one task is superior to another. Moreover, patients may prefer one task over the other. Similar issues exist in CBM-I, in which most studies have used the same scenario paradigm.

Questions also remain about the ideal stimuli. We do not know whether personalized stimuli are superior to standard stimuli sets. In addition, some studies have tested dynamic CBM protocols that begin with neutral stimuli and gradually become more positive over the course of several sessions [77]. Most multisession protocols presented the same stimuli in each session. It is unclear whether repeated practice with the same stimuli is superior to introducing new stimuli. The latter could potentially increase generalization of changes and patient engagement.

Future research may inform the treatment rationale provided to patients. It is currently unclear exactly what patients should be told regarding the purpose of CBM tasks and the task instructions. For example, Krebs et al. found that informing participants that the probe will always appear in the location of a particular type of word enhanced CBM-A's effects on attention and worry in nonanxious individuals [49]. However, MacLeod et al. found that providing explicit instructions diminished effects on anxiety [99]. In CBM-I, explicit instructions have typically not been provided. However, given the more explicit nature of these tasks, participants are very much aware that scenarios ended in either a positive or negative manner [69]. Moreover, participant knowledge of the scenarios' valence partially mediated the effect of training group on interpretation bias. Thus, it seems important to investigate how providing this explicit knowledge to participants prior to CBM-I may affect changes in interpretation and anxiety.

Finally, a principal future direction will be identifying appropriate therapeutic uses of CBM. Owing to CBM's unique features (e.g., standardized computerized delivery, no therapist contact, low demands on patients and brevity), there are a number of potential clinical uses. First, CBM has demonstrated promise as a stand-alone treatment for GSP and GAD. CBM as a stand-alone treatment would provide individuals with anxiety disorders with a new treatment option. Having more options may be beneficial for patients who do not respond to or cannot access current treatments, for patients or providers who find existing treatments unacceptable and for personalized medicine.

Cognitive bias modification may also have utility as an adjunctive intervention or as an option in stepped care treatment. It is possible that some patients may respond better to CBT if they also receive CBM. Should CBM improve attentional control, it may help patients better engage in exposures. It may assist those patients who get 'stuck' in cognitive restructuring by bypassing some of their automatic mental habits. Similarly, CBM could be an adjunct to pharmacotherapy. CBM may also prove useful in relapse prevention following treatment, as it directly targets cognitive vulnerabilities. Currently, we have no evidence regarding these potential uses of $\mathrm{CBM}$, and future research examining $\mathrm{CBM}$ as adjunctive treatment is needed.

An exciting potential application of CBM is in prevention. Studies have already demonstrated that CBM can buffer healthy and at-risk individuals from stressful situations $[45,46]$. Moreover, studies in children $[68,72,73]$ and adolescents $[100]$ demonstrate that cognitive biases are malleable in youth. At-risk children and adolescents are particularly important populations for CBM to target given that many anxiety disorders onset during this time. In addition, given that children and adolescent brains are developing, intervening during this period may enhance CBM's effects compared with attempting to modify longstanding mental habits in adults. 
Another issue in determining the best uses of CBM involves dissemination. Several studies have demonstrated that CBM can be delivered at home using online programs, which would enhance the accessibility of treatment. However, at-home protocols have not been tested in clinical samples, which need more attention and clinical monitoring. Preliminary data suggest that while patients appreciate the convenience of an at-home treatment, they also recognize the benefits of coming to an office (e.g., quiet place, scheduled time and social interaction) [85]. Should CBM be disseminated as a self-help intervention in the home, it will be important to determine whether CBM is better (i.e., more efficacious, easier to use and more acceptable) compared with existing self-help interventions (bibliotherapy and computerized CBT).

\section{Expert commentary}

Attention and interpretation biases are important treatment targets in anxiety disorders. Although existing treatments affect cognitive biases, CBM offers an alternative approach. CBM-A has shown preliminary efficacy for GSP and GAD in double-blind, placebocontrolled trials. If the current effect sizes are proven to be reliable, CBM-A should be considered as a treatment option for these disorders. Although CBM-I has been tested in many analogue samples, data from clinical populations are lacking. With the limited data in mind, CBM is a promising intervention with multiple implementation possibilities. As CBM targets transdiagnostic mechanisms, it is consistent with recent pushes toward unified treatments for anxiety and other emotional disorders. There are numerous areas ripe for future research to advance the field regarding CBM's efficacy, acceptability, mechanisms of action and utility as an adjunctive or stand-alone treatment.

\section{Five-year view}

Cognitive bias modification will continue to grow exponentially, both as a basic experimental psychopathology paradigm and as a treatment. There are a number of ongoing clinical trials from which data will be available in the next 5 years. For example, Amir and colleagues in San Diego (CA, USA) are completing RCTs of CBM-A for GAD delivered at home, CBM-A for veterans with post-traumatic stress disorder, and CBM-I for GSP. BarHaim and colleagues will have data from their large randomized trial of CBM-A in children diagnosed with anxiety disorders. We will continue to see an extension of CBM to new anxiety populations (e.g., OCD), other psychiatric populations (e.g., addictions and eating disorders) and applied as a transdiagnostic treatment. Ongoing trials examining the combination of CBM with existing therapies (e.g., CBT) will provide important data about CBM's utility as an adjunctive treatment. The next several years will reveal initial data on personalizing CBM to the individual, in terms of ideographic stimuli, predictors of response (e.g., readiness to acquire a cognitive bias [101], genetic matching and symptom severity) and treatment setting (e.g., primary care). Finally, CBM treatments will be made available directly to consumers. Indeed, one company recently began offering CBM-A software to consumers [201].

\section{Acknowledgments}

The author is grateful to Dr Nader Amir for his comments on a draft of this manuscript and to Hannah Boettcher for her assistance with the manuscript.

\section{References}

Papers of special note have been highlighted as:

- of interest 
1. Kessler RC, Berglund P, Demier O, Jin R, Merikangas KR, Walters EE. Lifetime prevalence and age-of-onset distributions of DSM-IV disorders in the National Comorbidity Survey Replication. Arch Gen Psychiatry. 2005; 62:593-602. [PubMed: 15939837]

2. Beard C, Weisberg RB, Keller MB. Health-related quality of life across the anxiety disorders: findings from a sample of primary care patients. J Anxiety Disord. 2010; 24:559-564. [PubMed: 20418054]

3. Stein MB, Heimberg RG. Well-being and life satisfaction in generalized anxiety disorder: comparison to major depressive disorder in a community sample. J Affect Disord. 2004; 79:161166. [PubMed: 15023490]

4. Kessler RC. The impairments caused by social phobia in the general population: implications for intervention. Acta Psychiatr Scand. 2003; 108:19-27.

5. Pinto A, Mancebo MC, Eisen JL, Pagano ME, Rasmussen SA. The Brown Longitudinal Obsessive Compulsive Study: clinical features and symptoms of the sample at intake. J Clin Psychiatry. 2006; 67:703-711. [PubMed: 16841619]

6. Yates WR. Phenomenology and epidemiology of panic disorder. Ann Clin Psychiatry. 2009; 21:95102. [PubMed: 19439159]

7. Katzelnick D, Greist JH. Social anxiety disorder: an unrecognized problem in primary care. J Clin Psychiatry. 2001; 62:11-16. [PubMed: 11206029]

8. Katzelnick DJ, Kobak KA, DeLeire T, et al. Impact of generalized social anxiety disorder in managed care. Am J Psychiatry. 2001; 158:1999-2007. [PubMed: 11729016]

9. Hoffman DL, Dukes EM, Wittchen HU. Human and economic burden of generalized anxiety disorder. Depress Anxiety. 2008; 25:72-90. [PubMed: 17146763]

10. Hoffman E, Mathew S. Anxiety disorders: a comprehensive review of pharmacotherapies. Mt Sinai J Med. 2008; 75:248-262. [PubMed: 18704983]

11. Blanco C, Schneier FR, Schmidt A, et al. Pharmacological treatment of social anxiety disorder: a meta-analysis. Depress Anxiety. 2003; 18:29-40. [PubMed: 12900950]

12. Mitte K. Meta-analysis of cognitive-behavioral treatments for generalized anxiety disorder: a comparison with pharmacotherapy. Psychol Bull. 2005; 131:785-795. [PubMed: 16187860]

13. Hofmann SG, Smits JAJ. Cognitive-behavioral therapy for adult anxiety disorders: a meta-analysis of randomized placebo-controlled trials. J Clin Psychiatry. 2008; 69:621-632. [PubMed: 18363421]

14. Weisberg RB, Dyck I, Culpepper L, Keller MB. Psychiatric treatment in primary care patients with anxiety disorders: a comparison of care received from primary care providers and psychiatrists. Am J Psychiatry. 2007; 164:276-282. [PubMed: 17267791]

15. Gunter RW, Whittal ML. Dissemination of cognitive-behavioral treatments for anxiety disorders: overcoming barriers and improving patient access. Clin Psychol Rev. 2010; 30:194-202. [PubMed: 19942331]

16•. Bar-Haim Y. Research review: attention bias modification (ABM): a novel treatment for anxiety disorders. J Child Psychol Psychiatry. 2010; 51(8):859-870. Excellent review of cognitive bias modification for attention (CBM-A). [PubMed: 20456540]

17. Browning M, Holmes EA, Harmer CJ. The modification of attentional bias to emotional information: a review of the techniques, mechanisms, and relevance to emotional disorders. Cogn Affect Behav Neurosci. 2010; 10:8-20. Excellent review of CBM-A. [PubMed: 20233952]

18. Hakamata Y, Lissek S, Bar-Haim Y, et al. Attention bias modification treatment: a meta-analysis toward the establishment of novel treatment for anxiety. Biol Psychiatry. 2010; 68:982-990. First meta-analysis of CBM-A for anxiety. [PubMed: 20887977]

19. Taylor, CT.; Amir, N. Attention and emotion regulation. In: Kring, AM.; Sloan, DM., editors. Emotion Regulation And Psychopathology: A Transdiagnostic Approach To Etiology And Treatment. Guilford Press; NY, USA: 2010. p. 380-404.

20. MacLeod C, Mathews A, Tata P. Attentional bias in emotional disorders. J Abnorm Psychol. 1986; 95:15-20. [PubMed: 3700842]

21. Bar-Haim Y, Dominique L, Pergamin L, Bakermans-Kranenburg MJ, van IJzendoorn MH. Threatrelated attentional bias in anxious and nonanxious individuals: a meta-analytic study. Psychol Bull. 2007; 133:1-24. [PubMed: 17201568] 
22. Macleod C, Hagan R. Individual differences in the selective processing of threatening information, and emotional responses to a stressful life event. Behav Res Ther. 1992; 30:151-161. [PubMed: 1567344]

23. van den Hout M, Tenney N, Huygens K, Merckelbach H, Kindt MR. Responding to subliminal threat cues is related to trait anxiety and emotional vulnerability: a successful replication of MacLeod and Hagan (1992). Behav Res Ther. 1995; 33:451-454. [PubMed: 7755532]

24. Fox E, Cahill S, Zougkou K. Preconscious processing biases predict emotional reactivity to stress. Biol Psychiatry. 2010; 67:371-377. [PubMed: 20113741]

25. Hirsch C, Mathews A. Interpretive inferences when reading about emotional events. Behav Res Ther. 1997; 35:1123-1132. [PubMed: 9465445]

26. Hirsch C, Mathews A. Impaired positive inferential bias in social phobia. J Abnorm Psychol. 2000; 109:705-712. [PubMed: 11195994]

27. Richards A, French CC. An anxiety-related bias in semantic activation when processing threat/ neutral homographs. Q J Exp Psychol A. 1992; 45A:503-525. [PubMed: 1308736]

28. Beard C, Amir N. Interpretation in social anxiety: when meaning precedes ambiguity. Cognit Ther Res. 2009; 33:406-415.

29. Roth D, Antony MM, Swinson RP. Interpretations for anxiety symptoms in social phobia. Behav Res Ther. 2001; 39:129-138. [PubMed: 11153968]

30. Hazlett-Stevens H, Borkovec TD. Interpretive cues and ambiguity in generalized anxiety disorder. Behav Res Ther. 2004; 42:881-892. [PubMed: 15178464]

31. Hirsch CR, Clark DM. Information processing bias in social phobia. Clin Psychol Rev. 2004; 24:799-825. [PubMed: 15501557]

32. Ouimet AJ, Gawronski B, Dozois DJA. Cognitive vulnerability to anxiety: a review and integrative model. Clin Psychol Rev. 2009; 29:459-470. [PubMed: 19552990]

33. Ehlers A, Mayou RA, Bryant B. Cognitive predictors of posttraumatic stress disorder in children: results of a prospective longitudinal study. Behav Res Ther. 2003; 41:1-10. [PubMed: 12488116]

34. Mayou RA, Ehlers A, Bryant B. Posttraumatic stress disorder after motor vehicle accidents: 3-year follow-up of a prospective longitudinal study. Behav Res Ther. 2002; 40:665-675. [PubMed: 12051485]

35. Coles ME, Horng B. A prospective test of cognitive vulnerability to obsessive-compulsive disorder. Cogn Ther Res. 2006; 30:723-734.

36. Abramowitz JS, Nelson CA, Rygwall R, Khandker M. The cognitive mediation of obsessivecompulsive symptoms: a longitudinal study. J Anxiety Disord. 2007; 21:91-104. [PubMed: 16806800]

37. Wells, A. Emotional Disorders and Metacognition: Innovative Cognitive Therapy. Wiley; NJ, USA: 2000.

38. Segal, ZV.; Williams, JMG.; Teasdale, JD. Mindfulness-Based Cognitive Therapy for Depression: A New Approach to Preventing Relapse. Guilford Press; NY, USA: 2002.

39. Mattia J, Heimber RH, Hope DA. The revised Stroop color-naming task in social phobics. Behav Res Ther. 1993; 31:305-313. [PubMed: 8476405]

40. Lundh L, Ost L. Attentional bias, self-consciousness and perfectionism in social phobia before and after cognitive-behaviour therapy. Scand J Behav Ther. 2001; 30:4-16.

41. Franklin ME, Huppert J, Langner R, Leiberg S, Foa EB. Interpretation bias: a comparison of treated social phobics, untreated social phobics, and controls. Cogn Ther Res. 2005; 29:289-300.

42. Legerstee JS, Tulen JH, Dierckx B, et al. CBT for childhood anxiety disorders: differential changes in selective attention between treatment responders and non-responders. J Child Psychol Psychiatry. 2010; 51:162-172. [PubMed: 19744254]

43. Koster EH, Fox E, MacLeod C. Introduction to the special section on cognitive bias modification in emotional disorders. J Abnorm Psychol. 2009; 118:1-4. Excellent introduction to cognitive bias modification. [PubMed: 19222308]

44. Dandeneau SD, Baldwin MW. The inhibition of socially rejecting information among people with high versus low self-esteem: the role of attentional bias and the effects of bias reduction training. J Social Clin Psychol. 2004; 23:584-602. 
45. Dandeneau SD, Baldwin MW, Baccus JR, Sakellaropoulo M, Pruessner JC. Cutting stress off at the pass: reducing vigilance and responsiveness to social threat by manipulating attention. J Pers Proc Ind Diff. 2007; 93:651-666.

46. See J, MacLeod C, Bridle R. The reduction of anxiety vulnerability through the modification of attentional bias: a real-world study using a home-based cognitive bias modification procedure. $\mathrm{J}$ Abnorm Psychol. 2009; 118:65-75. [PubMed: 19222315]

47•. MacLeod C, Rutherford E, Campbell L, Ebsworthy G, Holker L. Selective attention and emotional vulnerability: assessing the causal basis of their association through the experimental manipulation of attentional bias. J Abnorm Psychol. 2002; 111:107-123. Classic CBM-A study demonstrating changes in attention and anxiety. [PubMed: 11866165]

48. Browning M, Holmes EA, Murphy SE, Goodwin GM, Harmer CJ. Lateral prefrontal cortex mediates the cognitive modification of attentional bias. Biol Psychiatry. 2010; 67(10):919-925. [PubMed: 20034617]

49. Krebs G, Hirsch CR, Mathews A. The effect of attention modification with explicit versus minimal instructions on worry. Behav Res Ther. 2010; 48:251-256. [PubMed: 19926075]

50. MacLeod C, Soong LY, Rutherford EM, Campbell LW. Internet-delivered assessment and manipulation of anxiety-linked attentional bias: validation of a free-access attentional probe software package. Behav Res Methods. 2007; 39:533-538. [PubMed: 17958165]

51. Eldar S, Ricon T, Bar-Haim Y. Plasticity in attention: implications for stress response in children. Behav Res Ther. 2008; 46:450-461. [PubMed: 18313034]

52. Hayes S, Hirsch CR, Mathews A. Facilitating a benign attentional bias reduces negative thought intrusions. J Abnorm Psychol. 2010; 119:235-240. [PubMed: 20141260]

53. Eldar S, Bar-Haim Y. Neural plasticity in response to attention training in anxiety. Psychol Med. 2010; 40:667-677. [PubMed: 19627649]

54. Amir N, Weber G, Beard C, Bomyea J, Taylor CT. The effect of a single-session attention modification program on response to a public-speaking challenge in socially anxious individuals. $\mathrm{J}$ Abnorm Psychol. 2008; 117:860-868. [PubMed: 19025232]

55. Najmi S, Amir N. The effect of attention training on a behavioral test of contamination fears in individuals with subclinical obsessive-compulsive symptoms. J Abnorm Psychol. 2010; 119:136142. [PubMed: 20141250]

56. Reese HE, McNally RJ, Najmi D, Amir N. Attention training for reducing spider fear in spiderfearful individuals. J Anxiety Disord. 2010; 24:657-662. [PubMed: 20510577]

57. Li S, Tan J, Qian M, Liu X. Continual training of attentional bias in social anxiety. Behav Res Ther. 2008; 46:905-912. [PubMed: 18538305]

58. Hazen RA, Vasey MW, Schmidt NB. Attentional retraining: a randomized clinical trial for pathological worry. J Psychiatr Res. 2009; 43:627-633. [PubMed: 18722627]

59•. Schmidt NB, Richey JA, Buckner JD, Timpano KR. Attention training for generalized social anxiety disorder. J Abnorm Psychology. 2009; 118:5-14. First randomized controlled trial (RCT) of CBM-A for generalized social phobia.

60. Amir N, Beard C, Taylor C, et al. Attention training in individuals with generalized social phobia: a randomized controlled trial. J Consult Clin Psychol. 2009; 77:961-973. Second RCT of CBMA for generalized social phobia. [PubMed: 19803575]

61. Amir N, Beard C, Burns M, Bomyea J. Attention modification program in individuals with generalized anxiety disorder. J Abnorm Psychol. 2009; 118:28-33. First RCT of CBM-A for generalized anxiety disorder. [PubMed: 19222311]

62. Borkovec TD, Ruscio A. Psychotherapy for generalized anxiety disorder. J Clin Psychiatry. 2001; 62:37-45. [PubMed: 11414549]

63. Mathews A, Mackintosh B. Induced emotional interpretation bias and anxiety. J Abnorm Psychol. 2000; 109:602-615. Cognitive bias modification for interpretation study demonstrating changes in attention and anxiety. [PubMed: 11195984]

64. Grey S, Mathews A. Effects of training on interpretation of emotional ambiguity. Q J Exp Psychol A. 2000; 53:1143-1162. [PubMed: 11131817]

65. Hertel PT, Mathews A, Peterson S, Kintner K. Transfer of training emotionally biased interpretations. Appl Cogn Psychol. 2003; 17:775-784. 
66. Hirsch CR, Mathews A, Clark DM. Inducing an interpretation bias changes self-imagery: a preliminary investigation. Behav Res Ther. 2007; 45:2173-2181. [PubMed: 17178103]

67. Mackintosh B, Mathews A, Yiend J, Ridgeway V, Cook E. Induced biases in emotional interpretation influence stress vulnerability and endure despite changes in context. Behav Ther. 2006; 37:209-222. [PubMed: 16942973]

68. Muris P, Huijding J, Mayer B, Hameetman M. A space odyssey: experimental manipulation of threat perception and anxiety-related interpretation bias in children. Child Psychiatry Hum Dev. 2008; 39:469-480. [PubMed: 18446434]

69. Salemink E, van den Hout M, Kindt M. Trained interpretive bias and anxiety. Behav Res Ther. 2007; 45:329-340. [PubMed: 16859638]

70. Yiend J, Mackintosh B, Mathews A. Enduring consequences of experimentally induced biases in interpretation. Behav Res Ther. 2005; 43:779-797. [PubMed: 15890169]

71. Standage H, Ashwin C, Fox E. Is manipulation of mood a critical component of cognitive bias modification procedures? Behav Res Therapy. 2009; 48(1):4-10.

72. Vassilopoulos SP, Banerjee R, Prantzalou C. Experimental modification of interpretation bias in socially anxious children: changes in interpretation, anticipated interpersonal anxiety, and social anxiety symptoms. Behav Res Ther. 2009; 47(12):1085-1089. [PubMed: 19679299]

73. Muris P, Huijding J, Mayer B, Remmerswaal D, Vreden S. Ground control to Major Tom: experimental manipulation of anxiety-related interpretation bias by means of the 'space odyssey' paradigm and effects on avoidance tendencies in children. J Anxiety Disord. 2009; 23:333-340. [PubMed: 19201569]

74. Salemink E, van den Hout M, Kindt M. Trained interpretive bias: validity and effects on anxiety. J Behav Ther Exp Psychiatry. 2007; 38:212-224. [PubMed: 17141735]

75. Wilson EJ, MacLeod C, Mathews A. The causal role of interpretive bias in anxiety reactivity. J Abnorm Psychol. 2006; 115:103-111. [PubMed: 16492101]

76. Beard C, Amir N. A multi-session interpretation modification program: changes in interpretation and social anxiety symptoms. Behav Res Ther. 2008; 46:1135-1141. [PubMed: 18675400]

77. Hayes S, Hirsch CR, Krebs G, Mathews A. The effects of modifying interpretation bias on worry in generalized anxiety disorder. Behav Res Ther. 2010; 48:171-178. [PubMed: 19857859]

78. Hirsch CR, Hayes S, Mathews A. Looking on the bright side: accessing benign meanings reduces worry. J Abnorm Psychol. 2009; 118:44-54. [PubMed: 19222313]

79. Mathews A, Ridgeway V, Cook E, Yiend J. Inducing a benign interpretational bias reduces trait anxiety. J Behav Ther Exp Psychiatry. 2007; 38:225-236. [PubMed: 17141179]

80. Murphy R, Hirsch CR, Mathews A, Smith K, Clark DM. Facilitating a benign interpretation bias in a high socially anxious population. Behav Res Ther. 2007; 45:1517-1529. [PubMed: 17349970]

81. Steinman SA, Teachman BA. Modifying interpretations among individuals high in anxiety sensitivity. J Anxiety Disord. 2009; 24:71-78. [PubMed: 19747797]

82. Wilson EJ, MacLeod C, Mathews A, Rutherford EM. The causal role of interpretive bias in anxiety reactivity. J Abnorm Psychol. 2006; 115:103-111. [PubMed: 16492101]

83. Teachman BA, Addison LM. Training non-threatening interpretations in spider fear. Cogn Ther Res. 2008; 32:448-459.

84. Salemink E, van den Hout M, Kindt M. Effects of positive interpretive bias modification in highly anxious individuals. J Anxiety Disord. 2009; 23:676-683. [PubMed: 19272750]

85. Beard, C.; Weisberg, RB.; Perry, A.; Schofield, C.; Amir, N. Feasibility and acceptability of CBM in primary care settings. Presented at: World Congress of Behavioral and Cognitive Therapies; Boston, MA, USA. 2-5 June, 2010;

86. Salemink E, van den Hout M, Kindt MR. How does cognitive bias modification affect anxiety? Mediation analyses and experimental data. Behav Cogn Psychother. 2010; 38:59-66. [PubMed: 19995465]

87. Derryberry D, Reed MA. Anxiety-related attentional biases and their regulation by attentional control. J Abnorm Psychol. 2002; 111(2):225-236. [PubMed: 12003445]

88. Koster EH, Baert S, Bockstaele M, De Raedt R. Attentional retraining procedures: manipulating early or late components of attentional bias? Emotion. 2010; 10:230-236. [PubMed: 20364899] 
89. Grey SJ, Mathews AM. Cognitive bias modification - priming with an ambiguous homograph is necessary to detect an interpretation training effect. J Behav Ther Exp Psychiatry. 2009; 40:338343. [PubMed: 19249014]

90. Salemink E, van den Hout M. Trained interpretive bias survives mood change. J Behav Ther Exp Psychiatry. 2010; 41(3):310-315. [PubMed: 20304383]

91. Hoppitt L, Mathews A, Yiend J, Mackintosh B. Cognitive bias modification: the critical role of active training in modifying emotional responses. Behav Ther. 2009; 41(1):73-81. [PubMed: 20171329]

92. Holmes EA, Mathews A, Dalgleish T, Mackintosh B. Positive interpretation training: effects of mental imagery versus verbal training on positive mood. Behav Ther. 2006; 37:237-247. [PubMed: 16942975]

93. Kraemer HC, Mintz J, Noda A, et al. Caution regarding the use of pilot studies to guide power calculations for study proposals. Arch Gen Psychiatry. 2006; 63:484-489. [PubMed: 16651505]

94. Dandeneau SD, Baldwin MW. The buffering effects of rejection-inhibiting attentional training on social and performance threat among adult students. Contemp Educ Psychol. 2009; 34:42-50.

95. Harris LM, Menzies RG. Changing attentional bias: can it effect self-reported anxiety? Anxiety, Stress \& Coping. 1998; 11:167-179.

96. Salemink E, van den Hout M, Kindt M. Generalisation of modified interpretive bias across tasks and domains. Cognit Emot. 2010; 24:453-464.

97. Joorman J, Hertel PT, Lemoult J, Gotlib IH. Training forgetting of negative material in depression. J Abnorm Psychol. 2009; 118:34-43. [PubMed: 19222312]

98. Clerkin EM, Teachman BA. Training interpretation biases among individuals with symptoms of obsessive compulsive disorder. J Behav Ther Exp Psychiatr. 2011 (In press).

99. MacLeod, C.; Mackintosh, B.; Vujic, T. Does the explicit communication of the training contingency enhance the efficacy of CBM?. Presented at: Expert Meeting of CBM; Ghent, Belgium. May, 2009;

100. Lothmann C, Holmes EA, Chan SW, Lau JY. Cognitive bias modification training in adolescents: effects on interpretation biases and mood. J Child Psychol Psychiatry. 2010 (Epub ahead of print). 10.1111/j.1469-7610.2010.02286.x

101. Clarke P, MacLeod C, Shirazee N. Prepared for the worst: readiness to acquire threat bias and susceptibility to elevate trait anxiety. Emotion. 2008; 8:47-57. [PubMed: 18266515]

\section{Website}

201. Managing Your Anxiety. www.managingyouranxiety.com

Expert Rev Neurother. Author manuscript; available in PMC 2011 December 1. 


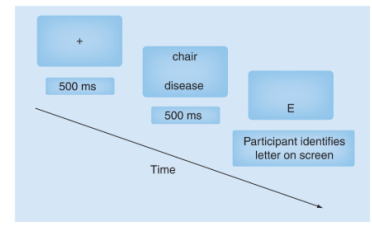

Figure 1. Example dot probe trial

Participants first see a fixation cross (+), followed by two stimuli (e.g., one threat word and one neutral word) and finally a probe (the letter ' $E$ ' or ' $F$ '). 


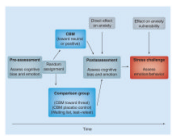

Figure 2. Cognitive bias modification study design for single-session experiment CBM: Cognitive bias modification. 


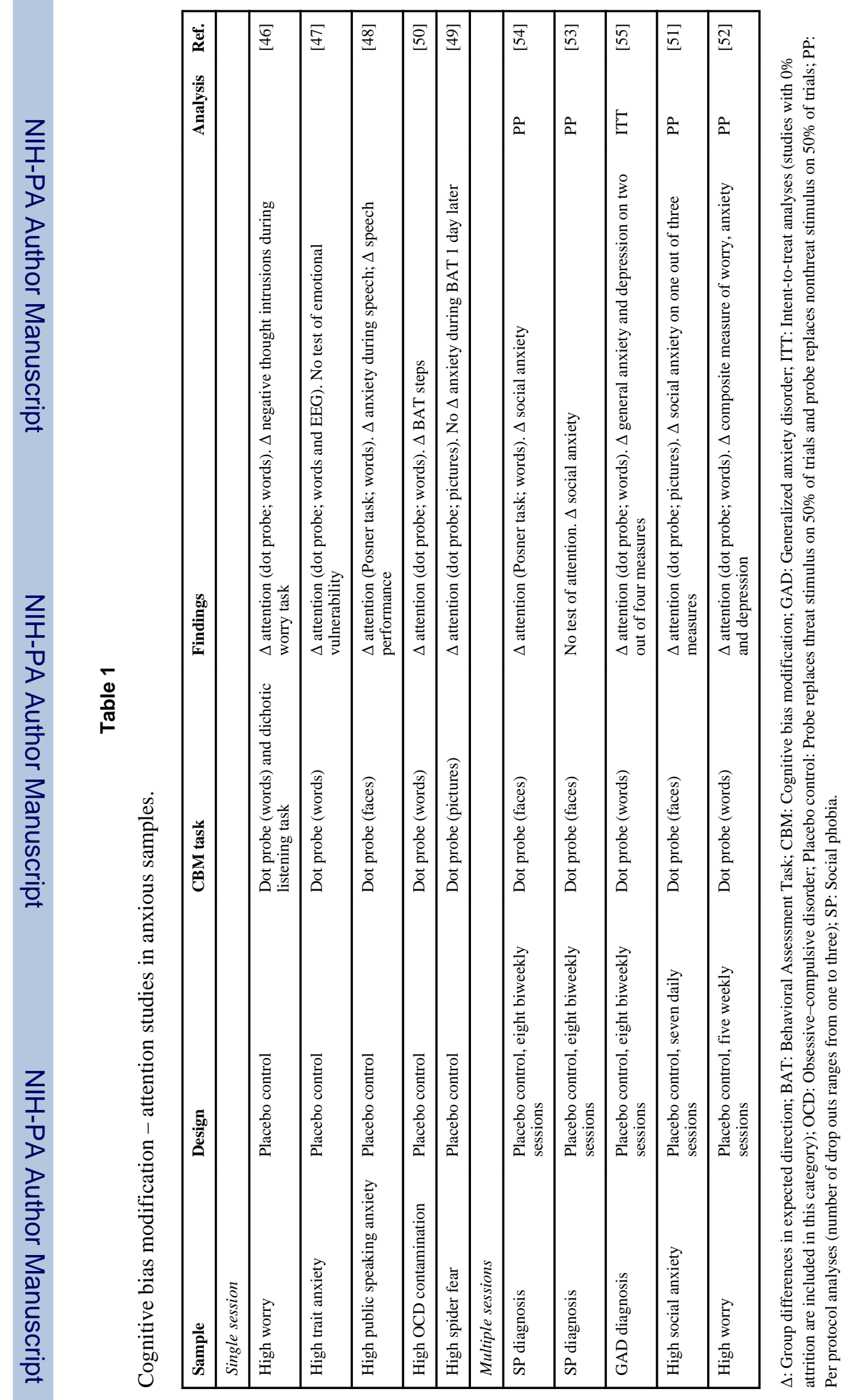

Expert Rev Neurother. Author manuscript; available in PMC 2011 December 1. 


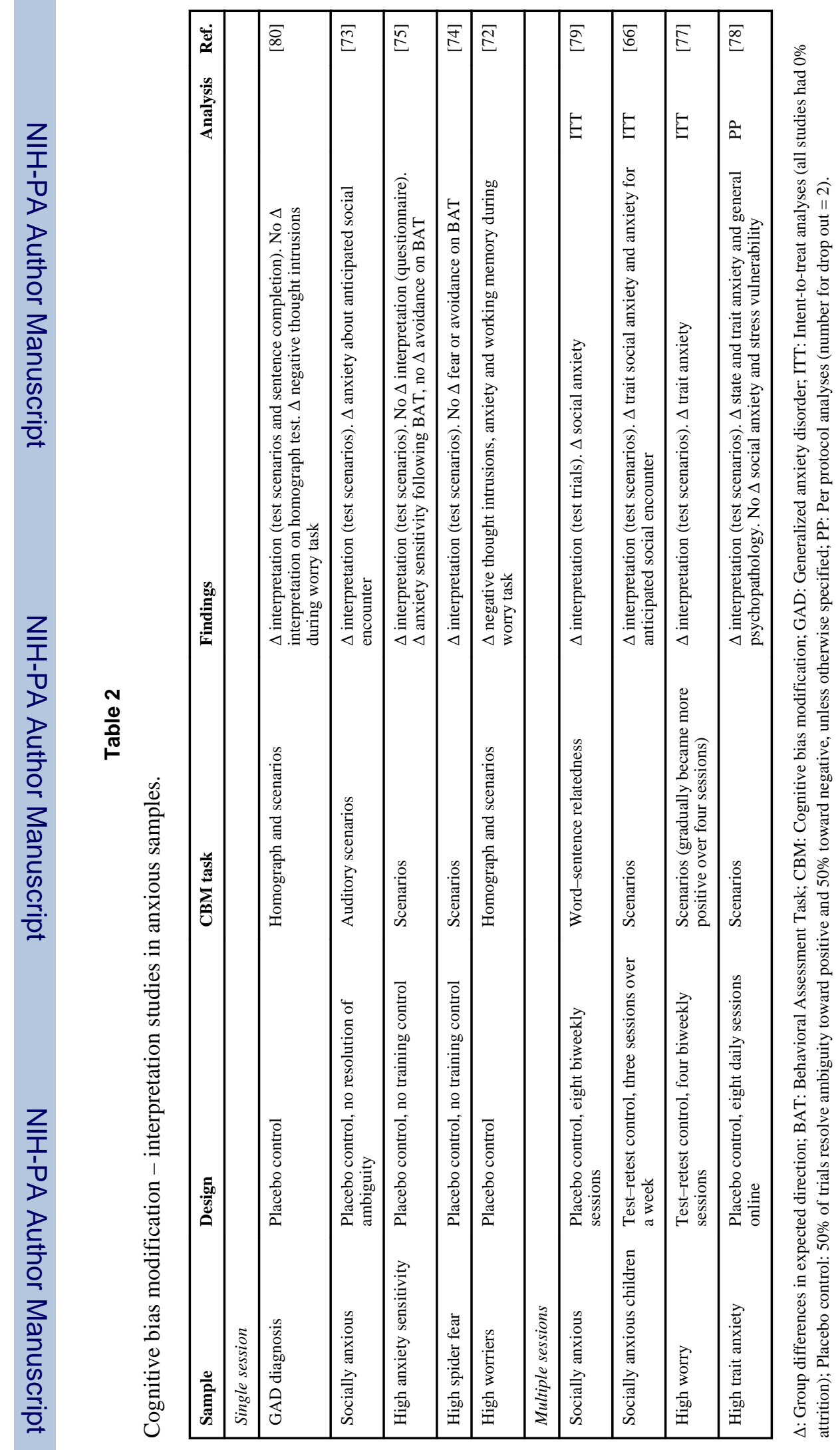

Expert Rev Neurother. Author manuscript; available in PMC 2011 December 1. 